\section{EUS-guided gallbladder drainage and hepatico- gastrostomy for acute cholecystitis and obstructive jaundice (with video)}
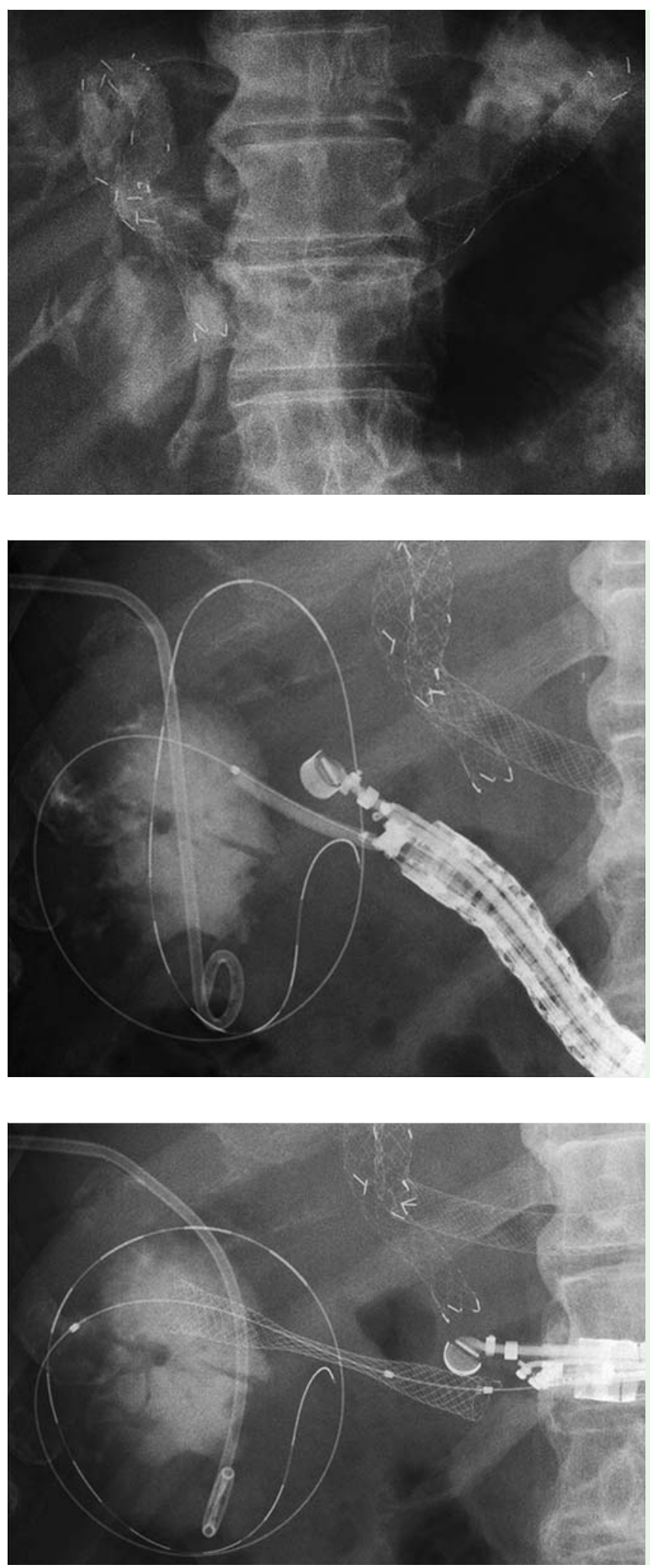

Fig. 1 Endoscopic ultrasound-guided hepaticogastrostomy using a fully covered metallic stent in a 71-year-old man with obstructive jaundice and acute cholecystitis.

Recently, endoscopic ultrasound (EUS)guided gallbladder drainage has been reported in patients who are unsuitable candidates for cholecystectomy $[1,2]$. However, this procedure can also lead to several complications, including stent migration. Novel methods or new devices are required to prevent these complications.

We report here the successful treatment of a patient with acute cholecystitis and obstructive jaundice complicated by advanced bile duct cancer, using EUS-guided hepaticogastrostomy and a novel technique of EUS-guided gallbladder drainage. A 71-year-old man was admitted to our hospital with obstructive jaundice and acute cholecystitis. Based on further evaluations, he was diagnosed histologically with unresectable bile duct cancer. Following failed endoscopic retrograde cholangiography, we performed EUS-guided hepaticogastrostomy ( $\bullet$ Fig. $\mathbf{1})$. The acute cholecystitis was treated by initial percutaneous gallbladder drainage (PTGBD), following which we successfully treated the condition. However, the acute cholecystitis recurred after removal of the PTGBD tube. Hence, we decided to performed EUS-guided gallbladder drainage. The gallbladder was punctured using a 19-G needle (SonoTip Pro Control 19G; Medi-Globe GmbH, Rosenheim, Germany) inserted via the duodenal bulb. Bile juice was aspirated and a small amount of contrast medium was injected. Next, a 0.025-inch guidewire (VisiGlide; Olympus Medical Systems, Tokyo, Japan) was inserted into the gallbladder. The gallbladder and duodenal wall were dilated using a $4 \mathrm{~mm}$ balloon catheter (Hurricane; Boston Scientific Japan, Tokyo, Japan) (O Fig.2). With a self-expandable metal stent (SEMS) inserted into the gallbladder $(10 \times 60 \mathrm{~mm}$ Wallstent; Boston Scientific Japan), the endoscope was carefully advanced and was released after passing the duodenal wall ( $\bullet$ Fig.3). Finally, to

Fig. 3 A fully covered metallic stent was inserted.

\section{Video 1}

Endoscopic ultrasound-guided hepaticogastrostomy using a fully covered metallic stent in a 71-year-old man with obstructive jaundice and acute cholecystitis. The gallbladder was punctured and contrast medium injected. Next, the guidewire was inserted and the fistula dilated using a balloon catheter. A fully covered metallic stent was placed and, finally, a pigtail plastic stent was placed into the metallic stent. 


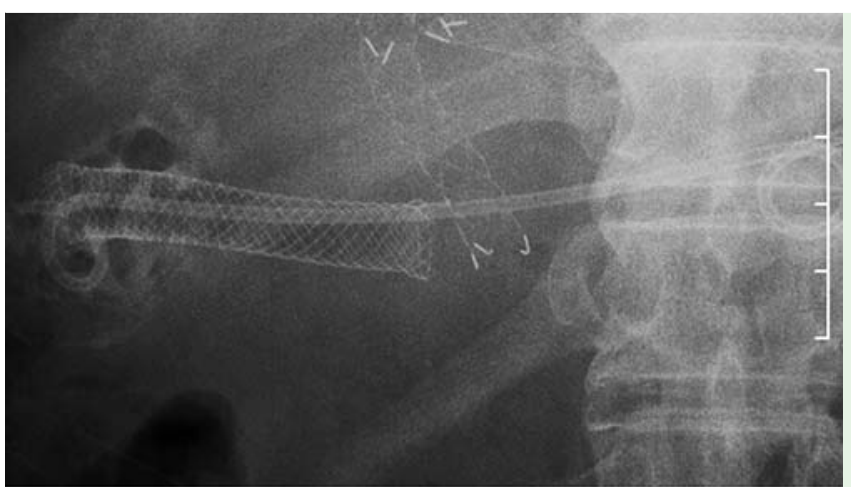

Fig.4 A pigtail plastic stent was placed inside the metallic stent.

avoid stent migration, we inserted a double pigtail plastic stent ( $7 \mathrm{Fr}, 10 \mathrm{~cm}$; Cook Medical, Bloomington, Indiana, USA) into the metallic stent ( $\bullet$ Fig. 4 ). Acute cholecystitis was not observed during 6 months after this procedure.

A novel metallic stent with flanges to prevent stent migration is now available for EUS-guided gallbladder drainage $[3,4]$, and this stent might be much better at preventing stent migration. However, if this flanged metallic stent is not used, we believe that a pigtail plastic stent should be added into the straight metallic stent, as in the present case, to avoid stent migration.
Endoscopy_UCTN_Code_TTT_1AT_2AB

Competing interests: None

Takeshi Ogura, Daisuke Masuda, Akira Imoto, Eiji Umegaki, Kazuhide Higuchi

Second Department of Internal Medicine, Osaka Medical College, Osaka, Japan

\section{References}

1 Itoi $T$, Itokawa F, Kurihara T. Endoscopic ultrasonography-guided gallbladder drainage: actual technical presentations and review of the literature (with video). J Hepatobiliary Pancreat Sci 2001; 18: 282-286
2 Itoi T, Binmoeller KF, Shah J et al. Clinical evaluation of novel lumen-apposing metal stent for endosonography-guided pancreatic pseudocyst and gallbladder drainage (with videos). Gastrointest Endosc 2012; 75: $870-876$

3 Turner BG, Rotam S, Paddu NU et al. Cholecystoduodenal drainage and gallstone removal in a patient with cholecystitis and unresectable cholangiocarcinoma. Endoscopy 2013; 45: E114-115

4 Mönkemüller K, Zabielski M, Didowacz-Grollmann $A$ et al. Endoluminal transgastric endoscopic anastomosis of the gallbladder using an anchoring self-expanding metal stent. Endoscopy 2013; 45: E164-166

\section{Bibliography}

DOI http://dx.doi.org/

10.1055/s-0033-1359135

Endoscopy 2014; 46: E75-E76

(c) Georg Thieme Verlag KG

Stuttgart · New York

ISSN 0013-726X

\section{Corresponding author}

\section{Takeshi Ogura, MD, PhD}

Second Department of Internal Medicine Osaka Medical College

2-7 Daigaku-machi, Takatsuki-shi

Osaka 569-8686

Japan

Fax: +81-726846532

oguratakeshi0411@yahoo.co.jp 